\title{
Acetyl Cholinesterase Inhibitors and Cell-Derived Peripheral Inflammatory Cytokines in Early Stages of Alzheimer's Disease
}

\author{
Nikolaos Kokras, PhD, *† Evangelia Stamouli, PhD, * Ioannis Sotiropoulos, PhD, $\$ \uparrow$ \\ Everina A. Katirtzoglou, MD, * Kostas T. Siarkos, MD, * Georgia Dalagiorgou, PhD, $\S$ \\ Krystallenia I. Alexandraki, PhD, $\neq / /$ Stavroula Coulocheri, PhD, $\neq$ \\ Christina Piperi, PhD, $\ddagger$ and Antonios M. Politis, PhD*
}

\begin{abstract}
:
Background: Clinical and preclinical studies firmly support the involvement of the inflammation in the pathogenesis of Alzheimer's disease (AD). Despite acetylcholinesterase inhibitors (AChEI) being widely used in $\mathrm{AD}$ patients, there is no conclusive evidence about their impact on the inflammatory response.

Methods: This study investigates peripheral proinflammatory cytokines (interferon gamma [IFN- $\gamma]$, tumor necrosis factor alpha [TNF- $\alpha]$, and interleukins $1 \beta[\mathrm{IL}-1 \beta]$ and 6 [IL-6]) by firstly comparing peripheral blood mononuclear cell (PBMC)-derived secretion in drug-naïve and AChEItreated $\mathrm{AD}$ patients versus healthy controls. A subset of those drug-naïve $\mathrm{AD}$ patients, who were prescribed the AChEI donepezil, was followed-up for 6 months to investigate if donepezil suppresses proinflammatory cellderived cytokine secretion.

Results: Patients with AD showed higher levels of PBMC-derived proinflammatory cytokines (IFN- $\gamma$, TNF- $\alpha$, IL-1 $\beta$, and IL-6) in comparison with healthy controls. On reexamination, previously drug-naïve $\mathrm{AD}$ patients who received donepezil treatment for 6 months displayed a decrease in cell-derived IFN- $\gamma$, TNF- $\alpha$, IL-1 $\beta$, and IL-6.

Conclusions: Proinflammatory PBMC-derived cytokines were increased in patients with $\mathrm{AD}$ in comparison with healthy controls and donepezilreduced proinflammatory cytokines when examining drug-naïve $\mathrm{AD}$ patients before and after AChEI treatment.
\end{abstract}

Key Words: Alzheimer's disease, inflammation, anticholinesterase inhibitors, cytokines, donepezil

(J Clin Psychopharmacol 2018;38: 00-00)

A zheimer's disease (AD), the most common type of dementia, is a progressive age-related neurodegenerative disorder clinically characterized by a gradual and progressive impairment in cognitive functions. Despite considerable progress in understanding the pathophysiology of the disease, ${ }^{1,2}$ AD remains a complex, multifactorial disorder with environmental, biological, and genetic risk factors. ${ }^{3}$ Interestingly, there is increasing evidence about the involvement of immune system abnormalities in $\mathrm{AD}$ pathology. ${ }^{4}$ Alzheimer's disease clinical and experimental studies suggest that

From the *First Department of Psychiatry, Eginitio Hospital, $\uparrow$ Department of Pharmacology, and $t$ Department of Biological Chemistry, Medical School, National and Kapodistrian University of Athens, Greece; §Life and Health Sciences Research Institute (ICVS), Medical School, University of Minho, \& ICVS/3B's-PT Government Associate Laboratory, Braga, Portugal; and |Department of Pathophysiology, Medical School, National and Kapodistrian University of Athens, Greece.

Received March 30, 2017; accepted after revision December 19, 2017.

Reprints: Nikolaos Kokras, PhD, Department of Pharmacology, Medical

School, National and Kapodistrian University of Athens, Greece, 75 Mikras

Asias St, 11527 Athens, Greece (e-mail: nkokras@med.uoa.gr).

N.K. and E.S. have equal contributions.

Copyright (C) 2018 Wolters Kluwer Health, Inc. All rights reserved.

ISSN: 0271-0749

DOI: $10.1097 / \mathrm{JCP} .0000000000000840$ cytokines-driven inflammatory pathways may trigger $\mathrm{AD}$ pathology and highlight their pathogenic role, as intense expression of these factors is found around $A \beta$ deposition areas and neurofibrillary tangles. ${ }^{5,6}$ Moreover, altered peripheral levels of interleukins $1 \beta$ (IL-1 $\beta)$, tumor necrosis factor alpha (TNF- $\alpha$ ), and interleukin (IL-6) are reported in patients with $\mathrm{AD}$ and higher blood serum IL-1 $\beta$ levels associate with a more rapid cognitive decline. ${ }^{7,8}$ Although measurements in peripheral inflammatory markers may only represent a partial reflection of brain pathology, peripheral cytokine levels correlate well with brain inflammation and their detection might be of use for disease assessment. ${ }^{9,10}$ Whereas evidence firmly supports the involvement of inflammation in the pathogenesis of $\mathrm{AD}$, there is still no conclusive evidence as to whether acetylcholinesterase inhibitors (AChEI) can have an impact on the inflammatory response. The AChEI are consistently used in AD patients as symptomatic therapy, mostly helping with cognitive and global outcome measures in a time-limited fashion. ${ }^{5}$ While experimental data suggest an AChEI anti-inflammatory effect on suppressing lymphocyte proliferation and proinflammatory cytokine production, ${ }^{11-13}$ a recent clinical study failed to identify any differences in IL1b, IL6, and TNFa after AChEI treatment. ${ }^{14}$ In this context, the present study investigated cell-derived proinflammatory cytokines by firstly comparing AChEI-treated and drug-naïve $\mathrm{AD}$ patients with healthy controls. We then prospectively followed-up a subset of those drug-naïve $\mathrm{AD}$ patients who were prescribed the AChEI donepezil to investigate the hypothesis that such treatment would suppress cell-derived proinflammatory cytokine secretion.

\section{METHODS}

\section{Patients}

The present clinical sample is part of a larger effort on investigating genetic associations in $\mathrm{AD}$, and further details of the patients' characteristics have been reported previously. ${ }^{15-17}$ For this study, 105 consecutive outpatients attending a psychogeriatric outpatient service at the Department of Psychiatry in Medical School of University of Athens were recruited. Informed consent was obtained from all patients, and the study was approved by the University of Athens Medical School Ethics Committee. Of them, 61 patients aged $75.9(\mathrm{SD}, 7.6)$ years with an average $\mathrm{AD}$ illness duration of 2.5 (SD, 2.0) years were not receiving AChEIs at the beginning of the study. Forty-four patients aged 73.9 (SD, 7.2) years and with an average illness duration of 4.0 (2.4) years were already under treatment with AChEIs. After informed consent, all patients were examined with the Structured Clinical Interview for the Diagnostic and Statistical Manual version IV Axis I Disorders (SCID). Thereafter, patients underwent a screening evaluation to determine if they met the following inclusion criteria: diagnosis of probable AD by National Institute of Neurological and 
Communicative Disorders and Stroke/Alzheimer's Disease and Related Disorders Association, ${ }^{18}$ Mini-Mental State Examination (MMSE) score of $\geq 10$, current residence at home, caregiver willing to accompany the participant to study visits, stable medical history and general health. Patients with a lifetime diagnosis of schizophrenia, bipolar disorder, or pre-AD anxiety disorder, current substance use disorder, acutely suicidal, or requiring inpatient psychiatric hospitalization were excluded from the study. Cognition was assessed using the MMSE, and the severity of dementia was assessed with the Clinical Dementia Rating (CDR) scale. ${ }^{19}$ Depressive symptoms were measured by the Greek version of the Cornell Scale for Depression in Dementia (CSDD) ${ }^{20}$ Additionally, 23 individuals aged 65.7 (SD, 6.9) years served as a control group. Those were randomly selected from the general population in Athens, Greece, with the following criteria: older than 60 years, MMSE score $>28$, normal physical examination, no history of neurological disease, and not meeting criteria for Diagnostic and Statistical Manual of Mental Disorders, version IV axis I diagnosis according to the World Health Organization Composite International Diagnostic Interview. For the follow-up study, of the 61 patients not receiving an AChEI at baseline assessment, 22 patients $(36 \%)$ were deemed suitable of being treated with donepezil $10 \mathrm{mg} / \mathrm{d}$. The treating physicians, who were blind to the specific aims, measurements, and outcomes of this study, were responsible to decide who of the original 61 patients would be treated with donepezil, based on their best clinical judgment and routine practices. There was no randomization to donepezil treatment and no influence of this study to the standard care the patients would receive. Those 22 patients received a target dose of $10 \mathrm{mg}$ daily, after a 6-week titration schedule for reducing cholinergic adverse events and were tested twice: at baseline, before treatment with donepezil, and at 6 months after treatment with $10 \mathrm{mg} / \mathrm{d}$ of donepezil.

\section{Biological Sampling and Assays}

Blood samples $(20 \mathrm{~mL})$ from all subjects were collected in heparin-containing tubes and were immediately processed using the Ficoll procedure. Briefly, $7 \mathrm{~mL}$ of peripheral blood collected in heparin-containing tubes was layered on top of a $7 \mathrm{~mL}$ FicollHypaque solution, according to manufacturers' instructions. After centrifugation, erythrocytes and granulocytes sedimented at the bottom of the tube and PBMCs (lymphocytes, monocytes) concentrated at the interface. By using aseptic techniques, the PBMCs were collected in separate tubes, washed, counted, and resuspended in $1.5 \mathrm{~mL}$ solution containing $80 \%$ fetal calf serum and $20 \%$ dimethyl sulfoxide. Cell viability as measured by trypan blue exclusion method always exceeded $95 \%$. Peripheral blood mononuclear cells $\left(1 \times 10^{6}\right.$ cells $\left./ \mathrm{mL}\right)$ containing Cryovials were stored in liquid nitrogen until analysis. For peripheral cytokines measurements, the enzyme-linked immunospot (ELISPOT) method was performed, which allows detection of real-time cytokine secretion by identifying the number of cells that actively secrete cytokines at a given time point with high sensitivity. ${ }^{21}$ Cytokines IL-1 $\beta$, TNF- $\alpha$, IL-6, and IFN- $\gamma$ were detected using a commercially available kit (Diaclone Elispot, Diaclone SAS, France). Briefly, well plates bottomed with polyvinylidene fluoride were coated with $25 \mu \mathrm{L} /$ well of $70 \%$ ethanol for 30 seconds at room temperature (RT) following and washed 3 times with $100 \mu \mathrm{L}$ (phosphate-buffered saline) PBS per well. Then, $100 \mu \mathrm{L}$ capturing antibody $(100-\mu \mathrm{L}$ antibody diluted in $10 \mathrm{~mL}$ PBS) was dispensed into each well and was incubated overnight at $+4^{\circ} \mathrm{C}$. Subsequently, wells were emptied, washed once with 100- $\mu$ L PBS, and incubated for 2 hours at RT with a blocking solution of $100 \mu \mathrm{L}$ Roswell Park Memorial Institute medium supplemented with $10 \%$ fetal calf serum. The plate was emptied and washed once with PBS. Next, $100 \mu \mathrm{L}$ of prepared cell suspension $\left(5 \times 10^{5}\right.$ cells $\left./ \mathrm{mL}\right)$ were dispensed into each well. In half of wells, the well-established mitogen PMA (phorbol 12-myristate 13-acetate, $200 \mathrm{ng} / \mathrm{mL}$ ) was added for activation of T lymphocytes to secrete cytokines and proliferate, as previously described. ${ }^{22}$ This cell density provided the clearest ELISPOT enumeration without background and optimum, nontoxic cell activity was obtained with $200 \mathrm{ng} / \mathrm{mL}$ PMA (tested concentrations $150-450 \mathrm{ng} / \mathrm{mL}$. The plate was again emptied, wells filled with $100 \mu \mathrm{L}$ PBS-T (PBS with $0.1 \%$ tween-20) for 10 minutes at $+4^{\circ} \mathrm{C}$ and washed 3 times with PBS-T. Subsequently, wells were filled with the detection antibody containing $1 \%$ bovine serum albumin and incubated for 90 minutes (RT). After incubation, the plate was washed with PBS-T and was incubated with streptavidin-alkaline phosphatase solution (1:5000, 1 hour, RT). After washing, wells were incubated with 5-bromo-4-chloro3-indolyl-phosphate/nitro blue tetrazolium buffer for 45 minutes. The reaction was stopped with distilled water, and the plate was dried and automatically analyzed using Immunospot Analyzer.

\section{Statistical Analysis}

Data were tested for normality and equality of variance and nonparametric testing was deemed more suitable and conservative. Comparisons between healthy subjects, AD patient without treatment, and $\mathrm{AD}$ patients under treatment with AChEIs were performed using the nonparametric Kruskal-Wallis (KW) test. Post-hoc multiple comparisons were performed according to Dunn's correction for type I error. Healthy subjects did not score at the CSDD, and data were analyzed by comparing AD patients with and without AChEI treatment using the 2-tailed nonparametric Mann-Whitney U test, taking ties into account. All data before and after treatment with AChEIs (donepezil) were tested using the 2-tailed non-parametric Wilcoxon/Pratt matched-pairs signed rank exact test. Statistical analysis was performed using the Graphpad Prism v.7.0b (GraphPad Software, La Jolla, CA). All values reported are mean $\pm(\mathrm{SD})$. Statistical significance was set at $P<0.05$.

\section{RESULTS}

\section{Cross-Sectional Study: Comparison of Drug-Naïve and AChEl-treated AD Patients With Healthy Controls}

As expected, healthy control subjects had higher MMSE scores than drug-naïve and under $\mathrm{AChEI}$ treatment $\mathrm{AD}$ patients $(\mathrm{KW}=53.12$; $P<0.0001$; post hoc, $P<0.0001, P<0.0001$, respectively) (Table 1 ). However, no difference was detected in the MMSE and CSDD mean scores between $\mathrm{AD}$ drug-naïve patients and those receiving an $\mathrm{AChEI}$ treatment. Interestingly, the CSDD median score of drug-naïve AD patients was on average 4 points higher than those already on an AChEI treatment $(\mathrm{U}=591, P=0.0028,95 \%$ confidence interval $[\mathrm{CI}]+0,+8$ points) (Table 1 ).

Regarding IFN- $\gamma$, there were significant differences among the 3 groups, either without or with PMA activation (KW $=56.19$, $P<0.0001 ; \mathrm{KW}=31.88, P<0.0001$; respectively). Healthy controls had lower counts of PBMC cells secreting IFN- $\gamma$ in comparison with drug-naïve $\mathrm{AD}$ patients with and without PMA activation $(P<0.0001$ and $P<0.0001$, respectively) and in comparison with AChEI-treated AD patients $(P<0.0148$ without PMA activation, $P<0.0001$ with PMA activation) (Fig. 1, A and B).

Tumor necrosis factor alpha was also significantly differentiated among the 3 groups, either without or with PMA activation $(\mathrm{KW}=56.06, P<0.0001 ; \mathrm{KW}=45.35, P<0.0001$; respectively). Healthy controls had lower levels of PBMC cells secreting TNF- $\alpha$, either without or with PMA activation than AChEI-treated and drug-naïve $\mathrm{AD}$ patients ( $P<0.0001$ for all 4 post-hoc comparisons) (Fig. 1, C and D). 
TABLE 1. Patient Characteristics, Demographics and Cell-Derived Cytokines for the Cross-sectional Part of the Study and the Follow-Up of Drug-Naïve Patients After 6 Months of Donepezil Treatment

\begin{tabular}{|c|c|c|c|c|c|}
\hline & \multicolumn{3}{|c|}{ Cross-Sectional Study } & \multicolumn{2}{|c|}{ Drug-Naïve AD Followed-Up } \\
\hline & Healthy Controls & AChEI-Treated AD & Drug-Naïve AD & Baseline & End Point \\
\hline $\mathrm{n}$ & 23 & 44 & 61 & \multicolumn{2}{|c|}{$22(36 \%$ of drug-naïve AD) } \\
\hline Females & $43 \%$ & $45 \%$ & $64 \%$ & \multicolumn{2}{|c|}{$77 \%$} \\
\hline Age & $65.74 \pm 6.99$ & $73.98 \pm 7.24$ & $75.87 \pm 7.59$ & \multicolumn{2}{|c|}{$75.73 \pm 4.94$} \\
\hline Illness duration & N/A & $4.02 \pm 2.41$ & $2.49 \pm 2.05$ & \multicolumn{2}{|c|}{$1.93 \pm 1.38$} \\
\hline AchEI mean dose & N/A & $6.59 \pm 2.53$ & 0 & 0 & $10 \mathrm{mg} /$ day \\
\hline MMSE & $28.83 \pm 1.23$ & $16.07 \pm 5.19$ & $18.43 \pm 7.67$ & $21.86 \pm 5.16$ & $19.05 \pm 3.84$ \\
\hline CSDD & N/A & $6.43 \pm 7.13$ & $7.28 \pm 5.95$ & $6.70 \pm 5.28$ & $3.47 \pm 3.98$ \\
\hline CDR early & N/A & $10 \%$ & $44 \%$ & $55 \%$ & $41 \%$ \\
\hline CDR mild & N/A & $45 \%$ & $26 \%$ & $27 \%$ & $41 \%$ \\
\hline CDR moderate & N/A & $38 \%$ & $20 \%$ & $14 \%$ & $9 \%$ \\
\hline CDR severe & N/A & $7 \%$ & $10 \%$ & $4 \%$ & $9 \%$ \\
\hline IFN- $\gamma$ & $210 \pm 81$ & $692 \pm 264^{*}$ & $1099 \pm 196^{*}$ & $1087 \pm 230$ & $968 \pm 243^{\dagger}$ \\
\hline IFN- $\gamma$ PMA & $713 \pm 283$ & $1068 \pm 378 *$ & $1386 \pm 461 *$ & $1388 \pm 591$ & $1192 \pm 324$ \\
\hline TNF- $\alpha$ & $286 \pm 124$ & $930 \pm 232 *$ & $1045 \pm 278^{*}$ & $1146 \pm 258$ & $941 \pm 222^{\dagger}$ \\
\hline TNF- $\alpha$ PMA & $720 \pm 201$ & $1272 \pm 367^{*}$ & $1489 \pm 605^{*}$ & $1733 \pm 768$ & $1246 \pm 268^{\dagger}$ \\
\hline IL-1 $\beta$ & $253 \pm 105$ & $760 \pm 565^{*}$ & $737 \pm 390^{*}$ & $946 \pm 257$ & $1007 \pm 197^{\dagger}$ \\
\hline IL-1 $\beta$ PMA & $548 \pm 150$ & $935 \pm 755^{*}$ & $908 \pm 543$ & $1041 \pm 288$ & $753 \pm 274^{\dagger}$ \\
\hline IL-6 & $301 \pm 99$ & $940 \pm 476^{*}$ & $1070 \pm 323 *$ & $977 \pm 357$ & $772 \pm 194$ \\
\hline IL-6 PMA & $781 \pm 343$ & $1125 \pm 531 *$ & $1393 \pm 564 *$ & $1254 \pm 556$ & $887 \pm 180^{\dagger}$ \\
\hline
\end{tabular}

All values are reported as means $\pm \mathrm{SD}$.

*Denotes a significant difference of drug-naïve or AChEI-treated AD patients vs health controls.

'Indicates a significant difference of AD patients after 6 months of AChEI treatment vs before treatment.

Similar differences were seen for IL-1 $\beta$, either without or with PMA activation $(\mathrm{KW}=23.89, P<0.0001 ; \mathrm{KW}=7.054$, $P<0.0294$; respectively). Healthy controls had lower counts of PBMC cells secreting IL-1 $\beta$ without or with PMA activation in comparison with drug-naïve AD patients $(P<0.0001$ and $P=0.0246$, respectively) and in comparison with AChEItreated AD patients without PMA activation only $(P=0.0006)$ (Fig. 1, E and F).

Regarding IL-6, differences were detected among the 3 groups, either without or with PMA activation $(\mathrm{KW}=37.93, P<0.0001$; $\mathrm{KW}=16.69, P<0.0001$; respectively). Healthy controls had fewer IL-6 secreting PBMC cells without or with PMA activation than untreated AD patients $(P<0.0001$ and $P<0.0001$, respectively), and fewer than AChEI-treated AD patients $(P<0.0001$ and $P=0.0299$, respectively) (Fig. $1, \mathrm{G}$ and $\mathrm{H}$ ).

Finally, drug-naïve and $\mathrm{AD}$ patients receiving an AChEI did not differ in TNF- $\alpha$, IL-1 $\beta$, and IL-6. However, AChEI-treated AD patients had lower IFN- $\gamma$ PBMC counts than drug-naïve AD patients $(P=0.0027)$ (Fig. 1A), but this difference was not observed after PMA stimulation (Fig. 1B).

\section{Follow-Up Study: Effect of an AChEl (Donepezil) Treatment on Drug-Naïve AD Patients}

Of the 61 drug-naïve $\mathrm{AD}$ patients presented, 22 patients (36\%) were deemed suitable by the treating physicians of being treated with donepezil $10 \mathrm{mg} / \mathrm{d}$ and they were tested twice: at baseline, before treatment with donepezil, and at follow-up, 6 months after treatment with $10 \mathrm{mg} / \mathrm{d}$ of donepezil.

Patients reexamined after a 6-month treatment with donepezil showed a significant reduction in the CSDD as indicated by the Wilcoxon matched-pairs 2-tailed signed rank test $(P=0.001)$, with a median difference of 4 points ( $95 \% \mathrm{CI},-6$ to 0 points). The CDR score after 6 months did not show any significant deterioration, as indicated by nonparametric testing, although a slight deterioration was observed in the MMSE score after 6 months (Table 1).

The analysis showed a reduction in the number of PBMC IFN- $\gamma$ secreting cells at baseline $(P=0.0244)$ (Fig. 2A) but not after PMA activation (Fig. 2B). Peripheral blood mononuclear cells secreting TNF- $\alpha$ were also reduced after donepezil treatment both before and after PMA $(P=0.0101$ and $P=0.0239$, respectively) (Fig. 2, C and D). Interleukins $1 \beta$ secreting cells were significantly reduced by donepezil treatment, as measured without and with PMA activation $(P=0.0009$ and $P=0.0203$, respectively) (Fig. 2, E and F). Finally, a reduction of cells secreting IL-6 was not evident without PMA stimulation (Fig. 2G), but after stimulation, the reduction in PBMCs secreting IL-6 levels was significant $(P=0.0268)$ (Fig. $2 \mathrm{H})$.

\section{DISCUSSION}

The current clinical study, based on patients with mainly early/mild $\mathrm{AD}$, demonstrates higher secretion of all monitored proinflammatory cytokines among AD patients. This is in line with previous evidence supporting the detrimental role of inflammatory cascades ${ }^{23}$ and demonstrating increased peripheral levels of proinflammatory cytokines such as TNF- $\alpha$, IL-1 $\beta$, and IL-6, as well as decreases in anti-inflammatory cytokines, such as IL-4 in patients with $\mathrm{AD}^{7,24,25}$ In contrast, negative results have also been reported in other human studies. ${ }^{26} \mathrm{~A}$ recent metaanalysis emphasized the variability of the peripheral cytokines in patients with $\mathrm{AD},{ }^{27}$ highlighting the difficulty of defining a direct link between peripheral (pro- and anti-) inflammatory cytokines and the etiology or response to treatment in such a multifactorial 
A

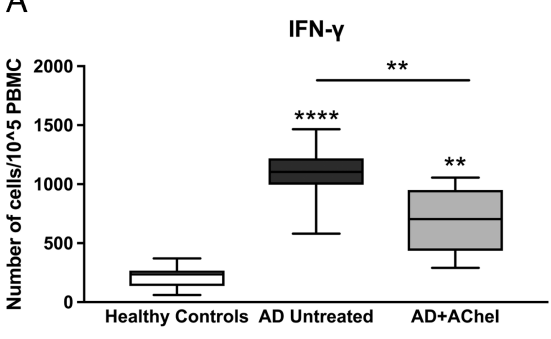

C

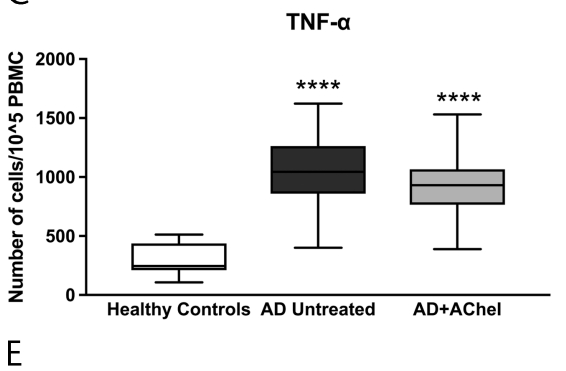

$\mathrm{E}$

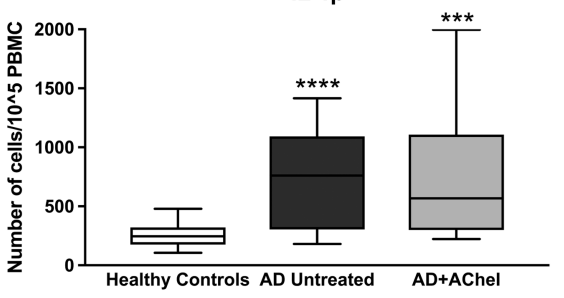

G

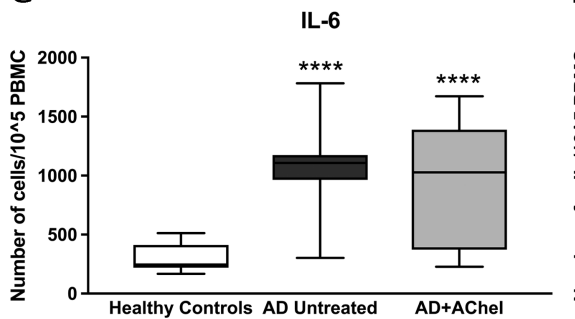

B

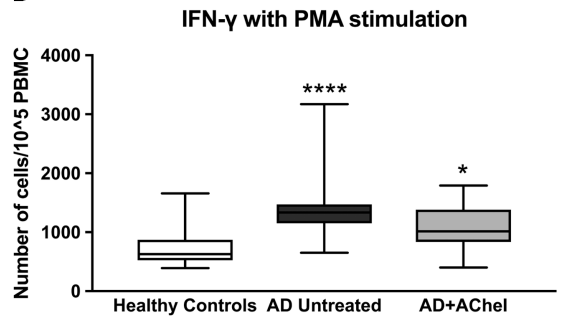

TNF- $\alpha$ with PMA stimulation

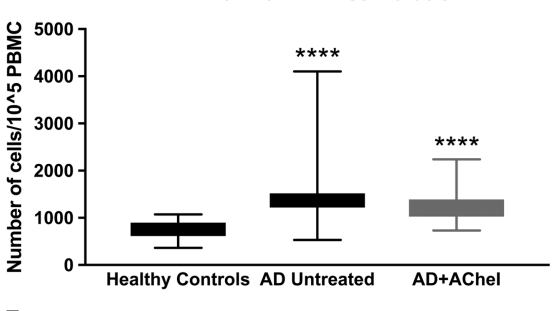

IL-1 $\beta$ with PMA stimulation

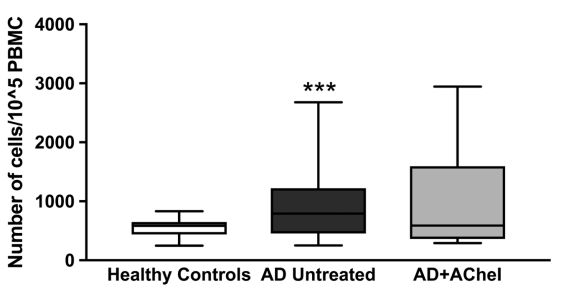

$\mathrm{H}$

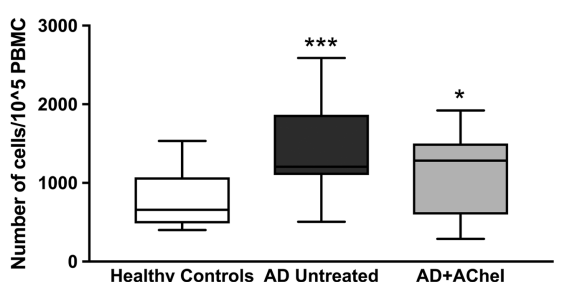

FIGURE 1. Number of cells in $10^{5}$ of PBMC secreting IFN- $y$, TNF- $\alpha$, IL-1 $\beta$, and IL- 6 . Data on the left side represent basal secretion and, on the right side, after Phorbol-12-Myristate-13-Acetate (PMA) stimulation. Two, 3, or 4 asterisks denote levels of significance $(P<0.01$, $P<0.001$, and $P<0.0001$, respectively) after nonparametric testing and post-hoc pairwise comparisons vs healthy controls. A pairwise comparison between treated and untreated AD patients is noted with a horizontal line.

disease where inflammatory cascades could be differently affected in early (presymptomatic) and late (postsymptomatic) stages of $\mathrm{AD}$. In contrast to monitoring of the aforementioned cytokines, very few studies have estimated systemic IFN- $\gamma$ in AD patients $9,28,29$ and their possible involvement in the neurodegenerative process in the brain. In previous studies, based on ELISA assays, IFN- $\gamma$ levels under basal conditions were either nondetectable ${ }^{28}$ or did not differ among groups of $\mathrm{AD}$ patients and healthy controls. ${ }^{29}$ However, a previous study showed that PBMC of patients with moderate/severe, but not early/mild, $\mathrm{AD}$ exhibit higher secretion of IFN- $\gamma$ when compared with healthy controls. ${ }^{9}$ In our study, we demonstrate a clear difference in IFN- $\gamma$ between patients with early/mild $\mathrm{AD}$ and healthy controls. The discrepancy between the current and previous studies could be attributed to the selected method of cytokines estimation, as ELISPOT might be more precise and accurate than ELISA in the detection of released cytokines.

On the other hand, there are conflicting reports on patients with $\mathrm{AD}$ regarding the effect of AChEIs on proinflammatory cytokines. Richardson et $\mathrm{al}^{14}$ found no AChEI-driven differences in IL-1 $\beta$, IL-6, and TNF- $\alpha$. On the contrary, the current study and previous studies showed that AChEI treatment reduced levels of PBMC-derived proinflammatory cytokines IL-1, IL-6, and TNF. ${ }^{24,30,31}$ A possible explanation for the above conflicting results among different clinical studies could be based on the different length of AChEI treatment as AChEIs apparently exert their action in a time-specific manner. In fact, while investigating the profile of the short-term and long-term use of AChEIs, a previous study detected a short-term AChEI effect on oxidative stress markers, which was not found at long-term treatment. ${ }^{32}$ Indeed, the current study shows that, while secretion of proinflammatory cytokines (including IFN- $\gamma$ ) was significantly higher in AD patients than healthy controls, 6-month donepezil treatment in patients with early/mild stage of $\mathrm{AD}$ results in a clear anti-inflammatory effect. However, despite this reduction, cytokines of donepezil-treated $\mathrm{AD}$ patients remained higher in comparison with healthy controls. A possible explanation is that 
A

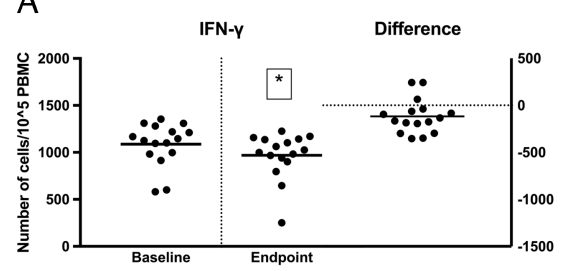

C

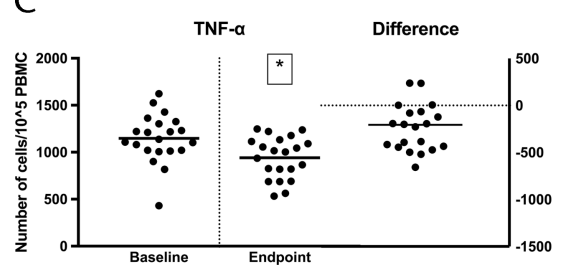

E

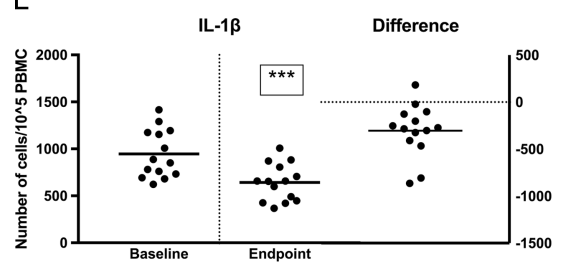

G

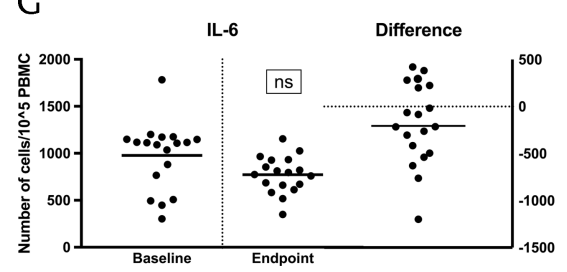

B

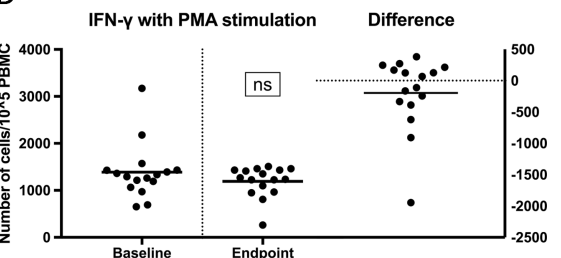

D

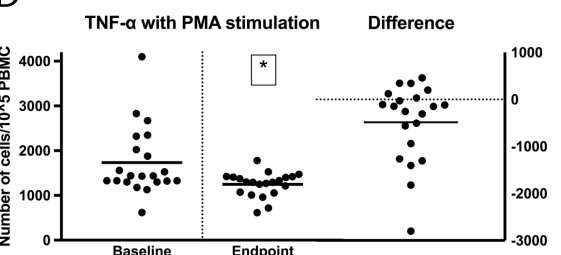

F

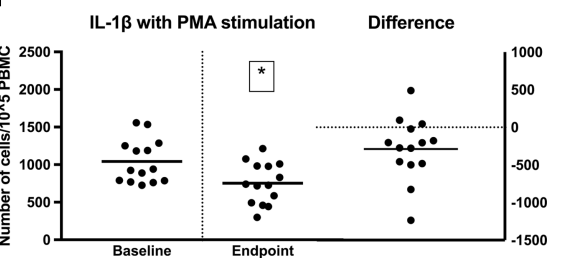

$\mathrm{H}$

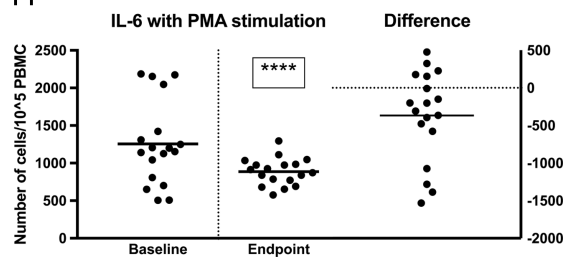

FIGURE 2. Effect of donepezil treatment on number of cells in $10^{5}$ of PBMCs secreting IFN-y, TNF- $\alpha$, IL-1 $\beta$, and IL-6. Baseline is before commencing treatment, and the end point is after 6 months of donepezil treatment. One, 3, or 4 asterisks denote levels of significance $(P<0.05, P<0.001$, and $P<0.0001$, respectively) calculated by Wilcoxon/Pratt nonparametric test. The left column represents basal secretion, and the right column contains data after PMA stimulation. On each pane, we report on the left values before (baseline) vs after treatment (end point) and the horizontal line represents the group mean at baseline and at the end point (6 months). On the right side of each pane, we depict the difference in absolute values for each patient to provide a better estimation of the effect of donepezil treatment on each patient. A horizontal line depicts the mean difference, and the thinner dashed line delineates the point of no difference after treatment.

the inflammatory-driven impact on $\mathrm{AD}$ neuronal malfunction/ damage might be a more prominent and irreversible process once neurodegeneration persists, in agreement with the hypothesis of an inflammatory endophenotype in AD patients. ${ }^{33}$ It should be mentioned that $80 \%$ of patients in this study were at the early/ mild stage of $\mathrm{AD}$, whereas only $20 \%$ at moderate/severe stage. A subgroup analysis, according to CDR staging, in a significantly larger sample would provide further insight to differences in PBMC-secreted proinflammatory cytokines according to the severity of $\mathrm{AD}$.

Interestingly, while their overall cognitive performance was not improved, AD patients receiving AChEI for 6 months exhibited an improvement in depression symptoms, as indicated by changes in the CSDD. Several studies have highlighted the potential role of inflammation as the connecting link between $\mathrm{AD}$ and depression. ${ }^{34}$ Patients with depression were also found with elevated proinflammatory cytokines, and common treatment targets have been proposed to protect from inflammation in $\mathrm{AD}$ and depression. ${ }^{35}$ A previous clinical study showed that donepezil treatment improved behavioral symptoms, including depression, ${ }^{36}$ while another study indicated that donepezil delayed more effectively the progression of cognitive impairment in those patients who were depressed at the beginning of donepezil treatment. ${ }^{37}$
Several confounding factors could influence the present result (such as "placebo" effect of treated patients or improvement of behavioral symptoms). Furthermore, there is no direct evidence regarding how the cytokine findings relate to the therapeutic effect (if any) of the AChEI. Moreover, the Ficoll-Hypaque method does not selectively identify lymphocytes versus monocytes as the source of cytokines and potential differences in these cells could influence the overall outcome, along with systemic diseases, infections, and inflammatory processes. However, all patients in this study where closely monitored and none displayed such events. In addition, the comparison of PBMC-derived cytokines in the prospective part of the study, where each patient is tested before and after treatment, provides additional evidence about the influence of AChEI treatment on overall. PBMC-derived cytokines levels.

In summary, this study shows a striking difference in proinflammatory cytokines, between controls versus drugnaive and AChEI-treated AD patients. Moreover, AChEI treatment, in this case donepezil, reduced proinflammatory cytokines when examining patients before and after treatment. Further studies are warranted to understand the role of such modulation of cytokines by AChEIs and its possible role in the progress of AD. 


\section{AUTHOR DISCLOSURE INFORMATION}

Funding was received from a program co-financed by the European Social Fund and the Greek State (GSRT LS5-3808). N.K. has received honoraria and travel support from JanssenCilag, Lundbeck, Sanofi-Aventis, Medochemie Generics, and Elpen S.A. None of those is relevant to this study.

The authors declare no conflicts of interest.

\section{REFERENCES}

1. Lyketsos CG, Szekely CA, Mielke MM, et al. Developing new treatments for Alzheimer's disease: the who, what, when, and how of biomarker-guided therapies. Int Psychogeriatr. 2008;20:871-889.

2. Lopes S, Vaz-Silva J, Pinto V, et al. Tau protein is essential for stress-induced brain pathology. Proc Natl Acad Sci U S A. 2016;113 E3755-E3763.

3. Sotiropoulos I, Cerqueira JJ, Catania C, et al. Stress and glucocorticoid footprints in the brain-the path from depression to Alzheimer's disease. Neurosci Biobehav Rev. 2008;32:1161-1173.

4. Calsolaro V, Edison P. Neuroinflammation in Alzheimer's disease: current evidence and future directions. Alzheimers Dement. 2016;12:719-732.

5. Rosenberg PB. Clinical aspects of inflammation in Alzheimer's disease Int Rev Psychiatry. 2005;17:503-514.

6. Wilcock DM. Neuroinflammatory phenotypes and their roles in Alzheimer's disease. Neurodegener Dis. 2014;13:183-185.

7. Angelopoulos P, Agouridaki H, Vaiopoulos H, et al. Cytokines in Alzheimer's disease and vascular dementia. Int J Neurosci. 2008;118: 1659-1672.

8. Holmes C, El-Okl M, Williams AL, et al. Systemic infection, interleukin 1 beta, and cognitive decline in Alzheimer's disease. J Neurol Neurosurg Psychiatry. 2003;74:788-789.

9. Huberman M, Shalit F, Roth-Deri I, et al. Correlation of cytokine secretion by mononuclear cells of Alzheimer patients and their disease stage. J Neuroimmunol. 1994;52:147-152.

10. Kaplin A, Carroll KA, Cheng J, et al. IL-6 release by LPS-stimulated peripheral blood mononuclear cells as a potential biomarker in Alzheimer's disease. Int Psychogeriatr. 2009;21:413-414.

11. Nizri E, Hamra-Amitay Y, Sicsic C, et al. Anti-inflammatory properties of cholinergic up-regulation: a new role for acetylcholinesterase inhibitors. Neuropharmacology. 2006;50:540-547.

12. Jiang Y, Zou Y, Chen S, et al. The anti-inflammatory effect of donepezil on experimental autoimmune encephalomyelitis in C57 BL/6 mice. Neuropharmacology. 2013;73:415-424.

13. Lataro RM, Silva CA, Tefe-Silva C, et al. Acetylcholinesterase inhibition attenuates the development of hypertension and inflammation in spontaneously hypertensive rats. Am J Hypertens. 2015;28:1201-1208.

14. Richardson C, Gard PR, Klugman A, et al. Blood pro-inflammatory cytokines in Alzheimer's disease in relation to the use of acetylcholinesterase inhibitors. Int J Geriatr Psychiatry. 2013;28: 1312-1317.

15. Olgiati P, Politis A, Albani D, et al. Effects of SORL1 gene on Alzheimer's disease. Focus on gender, neuropsychiatric symptoms and pro-inflammatory cytokines. Curr Alzheimer Res. 2013;10:154-164.

16. Politis A, Olgiati P, Malitas P, et al. Vitamin B12 levels in Alzheimer's disease: association with clinical features and cytokine production. J Alzheimers Dis. 2010;19:481-488.

17. Serretti A, Olgiati P, Politis A, et al. Lack of association between interleukin-1 alpha rs1800587 polymorphism and Alzheimer's disease in two Independent European samples. J Alzheimers Dis. 2009;16:181-187.

18. McKhann G, Drachman D, Folstein M, et al. Clinical diagnosis of Alzheimer's disease: report of the NINCDS-ADRDA Work Group under the auspices of Department of Health and Human Services Task Force on Alzheimer's Disease. Neurology. 1984;34:939-944.

19. Morris JC. The Clinical Dementia Rating (CDR): current version and scoring rules. Neurology. 1993;43:2412-2414.

20. Politis A, Maillis A, Thomadaki O, et al. Validity and reliability of the newly translated Cornell Scale for Depression in Dementia (CSDD) applied to Greek outpatients with depression and depression and Alzheimer's disease. Ann Gen Psychiatry. 2006;5:1.

21. Guerkov RE, Targoni OS, Kreher CR, et al. Detection of low-frequency antigen-specific IL-10-producing CD4(+) T cells via ELISPOT in PBMC: cognate vs. nonspecific production of the cytokine. J Immunol Methods. 2003;279:111-121.

22. Baran J, Kowalczyk D, Ozog M, et al. Three-color flow cytometry detection of intracellular cytokines in peripheral blood mononuclear cells: comparative analysis of phorbol myristate acetate-ionomycin and phytohemagglutinin stimulation. Clin Diagn Lab Immunol. 2001;8: 303-313.

23. Birch AM, Katsouri L, Sastre M. Modulation of inflammation in transgenic models of Alzheimer's disease. J Neuroinflammation. 2014;11:25

24. Reale M, Iarlori C, Gambi F, et al. Treatment with an acetylcholinesterase inhibitor in Alzheimer patients modulates the expression and production of the pro-inflammatory and anti-inflammatory cytokines. J Neuroimmunol. 2004; 148:162-171.

25. Reale M, Di Nicola M, Velluto L, et al. Selective acetyl- and butyrylcholinesterase inhibitors reduce amyloid- $\beta$ ex vivo activation of peripheral chemo-cytokines from Alzheimer's disease subjects: exploring the cholinergic anti-inflammatory pathway. Curr Alzheimer Res. 2014;11: 608-622.

26. Choi C, Jeong JH, Jang JS, et al. Multiplex analysis of cytokines in the serum and cerebrospinal fluid of patients with Alzheimer's disease by color-coded bead technology. J Clin Neurol. 2008;4:84-88.

27. Swardfager W, Lanctot K, Rothenburg L, et al. A meta-analysis of cytokines in Alzheimer's disease. Biol Psychiatry. 2010;68:930-941.

28. Rota E, Bellone G, Rocca P, et al. Increased intrathecal TGF-beta1, but not IL-12, IFN-gamma and IL-10 levels in Alzheimer's disease patients. Neurol Sci. 2006;27:33-39.

29. Singh VK, Guthikonda P. Circulating cytokines in Alzheimer's disease. J Psychiatr Res. 1997;31:657-660.

30. Gambi F, Reale M, Iarlori C, et al. Alzheimer patients treated with an AchE inhibitor show higher IL-4 and lower IL-1 beta levels and expression in peripheral blood mononuclear cells. J Clin Psychopharmacol. 2004;24: 314-321.

31. Reale M, Iarlori C, Gambi F, et al. Acetylcholinesterase inhibitors effects on oncostatin-M, interleukin-1 beta and interleukin-6 release from lymphocytes of Alzheimer's disease patients. Exp Gerontol. 2005;40: 165-171.

32. Sinem F, Dildar K, Gokhan E, et al. The serum protein and lipid oxidation marker levels in Alzheimer's disease and effects of cholinesterase inhibitors and antipsychotic drugs therapy. Curr Alzheimer Res. 2010;7:463-469.

33. Wyss-Coray T. Inflammation in Alzheimer disease: driving force, bystander or beneficial response? Nat Med. 2006;12:1005-1015.

34. Leonard BE. Inflammation, depression and dementia: are they connected? Neurochem Res. 2007;32:1749-1756.

35. Caraci F, Copani A, Nicoletti F, et al. Depression and Alzheimer's disease: neurobiological links and common pharmacological targets. Eur J Pharmacol. 2010;626:64-71.

36. Gauthier S, Feldman H, Hecker J, et al. Efficacy of donepezil on behavioral symptoms in patients with moderate to severe Alzheimer's disease. Int Psychogeriatr. 2002;14:389-404.

37. Lu PH, Edland SD, Teng E, et al. Donepezil delays progression to AD in MCI subjects with depressive symptoms. Neurology. 2009;72:2115-2121. 